Research Paper:

\title{
Revisiting the Urge to Operate: One-year Neurophysiological Follow-up in Severe CTS
}

\author{
Alireza Ashraf $^{1}$ (D) , Zahra Hooshanginezhad ${ }^{2}$ (D) Attiyeh Vasaghi ${ }^{3}$ (D) Nima Derakhshan ${ }^{4^{*}}$ (D)
}

1. MD., Professor, Department of Physical Medicine and Rehabilitation, Shiraz University of Medical Sciences, Shiraz, Iran 2. MD., Student Research Committee, Shiraz University of Medical Sciences, Shiraz, Iran

3. MD., Department of Physical Medicine and Rehabilitation, Shiraz University of Medical Sciences, Shiraz, Iran

4. Assistant Professor, Department of Neurosurgery, Shiraz University of Medical Sciences, Shiraz, Iran

$\begin{aligned} & \text { Use vour device to scan } \\ & \text { and read the article online }\end{aligned}$
Cltation Ashraf AR, Hooshanginezhad Z, Vasaghi A, Derakhshan N. Revisiting the Urge to Operate: One-Year Neurophysi-
ological Follow-up in Severe CTS. Iran J Neurosurg. 2020; 6(1):21-28. http://dx.doi.org/10.32598/irjns.6.1.4

\section{(c) (i) (8)}

Article info:

Received: 05 Sep 2019

Accepted: 10 Dec 2019

Available Online: 01 Jan 2020

Keywords:

Electrodiagnosis, Carpal tunnel syndrome, Boston questionnaire, Conservative treatment, Sensory Nerve Action

\section{ABSTRACT}

Background and Aim: Several approaches have been proposed for the treatment of Carpal Tunnel Syndrome (CTS) based on its severity. The aim of this study is to determine the optimal management for severe CTS.

Methods and Materials/Patients: This cross-sectional study was conducted for 22 months from August 2017 to June 2019 in a referral rehabilitation clinic in southern Iran. A total of 117 hands diagnosed with severe CTS were included in 78 patients according to Electrodiagnostic Study (EDX) reports (considering Sensory Proximal Latency (SPL) $>3.6 \mathrm{msec}$, Sensory Distal Latency (SDL) $>5.3 \mathrm{msec}$, Sensory Nerve Conduction Velocity (SNCV) $<30 \mathrm{~m} / \mathrm{sec}$, Distal Motor Latency (DML) $>6.5 \mathrm{msec}$ as being severe) who refused to undergo surgery. Boston questionnaire was filled out and conventional EDX was carried out at the first and the 1-year follow-up visits.

Results: In patients with a detectable Sensory Nerve Action Potential (SNAP), motor amplitude $(P<0.002)$ and latency $(\mathrm{P}<0.01)$, SPL $(\mathrm{P}<0.003)$ and SNCV $(\mathrm{P}<0.006)$, and Boston parameters improved significantly in the one-year follow-up visit compared with the results at the first visit. However, improvement in patients with absent or low amplitude SNAP at the first visit was only observed in proximal sensory latency $(P<0.005)$ and amplitude $(P<0.003)$.

Conclusion: There is a considerable chance for non-surgical improvement of patients with severe CTS in terms of symptom relief, hand function, and EDX parameters in those with detectable SNAP at the first visit; however, patients with undetectable SNAP have little, if any, the chance for improvement with conservative measures.

\section{* Corresponding Author:}




\section{Highlights}

- Many authorities consider surgical release as the only treatment for patients with severe CTS

- We believe that patients with severe CTS who have detectable median SNAPs have a substantial chance for recovery without surgery.

- The surgical release should be considered early in the management of patients with severe CTS and undetectable SNAPS

\section{Plain Language Summary}

Carpal tunnel syndrome is a common condition caused by entrapment of the median nerve in the wrist. Electrodiagnostic tests provide a severity scale which clarifies the treatment plan. Patients with mild and moderate CTS usually recover with non-surgical measures such as injections, bracing, and physical therapy. Even though many authorities consider surgery the only treatment for severe CTS, we believe that a substantial number of such patients who have detectable median SNAPs can defer surgery. Those with undetectable median SNAPs should consider early surgery because they do not have any chance for recovery with conservative modalities.

\section{Introduction}

arpal Tunnel Syndrome (CTS) is the most common entrapment neuropathy in upper extremities. The median nerve should pass through the carpal tunnel at the wrist to enter the hand, where it is surrounded by transverse carpal ligament superiorly and carpal bones at volar, medial and lateral aspects [1]. Median nerve compression in the tunnel might lead to ischemia, demyelination, and even axonal loss.

This entrapment neuropathy is commonly seen in females with the peak incidence in the 4th-6th decades of life. Patients with CTS present with hand numbness, tingling, pain, and even weakness of the hand, especially in the median nerve territory [2]. Although physical examination and specific tests provide reliable means for diagnosis, an electrodiagnostic study (EDX) provides quantitative information to confirm the diagnosis and to assess the treatment measures.

According to the Nerve Conduction Study (NCS) as a sensitive and specific test, CTS is classified as mild, moderate, and severe $[1,3]$. Based on the NCS grading, various treatment options are offered, such as surgical decompression, splinting, steroid injection, and physiotherapy. Surgical decompression is often considered for those with severe CTS and those who failed in improvement with conservative measures. Considering the potential complications of surgery such as palmar branch injury, infection, persistent pain, and wrist stiffness, conservative treatment is considered as a first choice in many instances of severe CTS [4].
However, several debates still exist in the optimal management of severe CTS that is still a matter of debate considering the promising results of surgical decompression in providing long-term relief of symptoms of CTS and improvement of NCS parameters [5-7].

Herein, utilizing EDX and Boston questionnaire, we followed the patients sustaining severe CTS who refused to undergo surgical treatment. We determined EDX parameters at the first visit to be useful in differentiating individuals who still have a chance with conservative treatment versus those who will benefit from early consideration of surgery.

\section{Methods and Materials/Patients}

\subsection{Study population}

This study was conducted in a cross-sectional fashion, during 22 months from August 2017 to June 2019 in Physical Medicine and Rehabilitation Clinic of Shahid Faghihi Hospital, a referral rehabilitation clinic, affiliated with Shiraz University of Medical Sciences in southern Iran.

All of the patients participated in the study after obtaining written informed consent. Their urgent need to undergo surgery and the disease consequences such as hand weakness and severe numbness were described for them. We included patients with severe CTS according to EDX parameters (Sensory Proximal Latency $(\mathrm{SPL})>3.6$, Sensory Distal Latency $(\mathrm{SDL})>5.3$, Sensory Nerve Conduction Velocity (SNCV) $<30$, Motor Distal Latency (MDL) >6.5) and symptoms such as paresthesia, pain, and numbness who were not willing to undergo 
surgery (due to costs, fear of complication, etc.). Patients who had secondary CTS due to pregnancy, diabetic mellitus, hypothyroidism, rheumatoid diseases such as lupus, scleroderma, and rheumatoid arthritis were excluded. Moreover, those with the wrist trauma, deformity, or surgical release of the median nerve were excluded. Seventy-nine patients (with 120 hands suffering CTS) aged between 28 and 84 participated in the study.

\subsection{EDX and BOSTON Questionnaire}

EDX was done for all the patients in the first visit. For the EDX study, hands' temperature was measured to be 31-32 ${ }^{\circ} \mathrm{C}$. Both median and ulnar nerves were assessed to rule out other neuropathies. The Compound Muscle Action Potentials (CMAP) were recorded from Abductor Pollicis Brevis (APB) and Abductor Digiti Minimi (ADM). All CMAPs stimulations were supramaximal. The action potential of APB was recorded by a surface electrode, $8 \mathrm{~cm}$ distal to the stimulation cathode, while ADM was excited $8 \mathrm{~cm}$ proximal to the recorder. Sensory Nerve Action Potentials (SNAP) were obtained from the 3rd and 5th digits antidromically. Median nerve SNAPs were obtained from the mid palm and the $3 \mathrm{rd}$ digit, where the recorder was placed $7 \mathrm{~cm}$ and $14 \mathrm{~cm}$ distal to the stimulating cathode, respectively. Ulnar nerve SNAPs were recorded $14 \mathrm{~cm}$ distal to the stimulator. Prolonged median nerve Sensory Proximal Latency (SPL) more than 3.6 msec with motor distal latency (MDL) over $6.5 \mathrm{msec}$, low amplitude or absent CMAP were considered "severe" CTS.

During the same visit, all patients were also asked to fill the self-administered Boston Questionnaire (BQ) which included 11 questions about the severity of symptoms and 8 questions about the functional status. Each answer scored the severity grading as 1 (healthy) to 5 (severe symptom or inability) $[5,8]$.The Persian translation of $\mathrm{BQ}$ was found to have acceptable reliability and validity in previous studies $[9,10]$.

\subsection{Outcome measures}

The main outcome measures of this study were the EDX parameters and BOSTON questionnaire grading of patients with severe CTS, compared in the first visit and the one-year follow-up visit.

\subsection{Statistical analysis}

In order to have $95 \%$ power to detect $1 \%$ difference between main outcome measures confidence limit $(Z=2)$ with Standard Deviation (SD) $=4$ and power to detect $1 \%$ difference between main outcome measures, we required 64 hands with severe CTS. $n=\left(\frac{(\delta \times Z)}{d}\right)^{2}$

Statistical analysis was performed using SPSS (Statistical Package for Social Sciences, Inc., Chicago, Illinois, USA) version 22. Paired t-test was used to compare the results of BQ and NCS parameters. Data were reported as mean (standard deviation), mean difference $\pm 95 \%$ Confidential Interval ( $\mathrm{Cl}$ ), and a two-sided p-value of less than 0.05 was considered to be statistically significant.

\section{Results}

All patients were asked to return for the second visit after 1 year. Considering the number of the patients being lost to follow-up, unavailability due to changes in address and phone number and the patients' preference to quit the study, 60 hands of 39 patients were eligible to be enrolled in the study.

Of the 60 hands enrolled in the second visit, 50 hands $(83.3 \%)$ and 10 hands (16.7\%) belonged to the women and men, respectively. The mean age of the patients was $50 \pm 10.6$ years. Twenty-one patients $(53.8 \%)$ had bilateral CTS, while 11 (28.2\%) had unilateral entrapment in their dominant hand, and $7(17.9 \%)$ had CTS in their non-dominant hand

For analysis of the Boston questionnaire, we subdivided the questions into symptoms and functional status, with 11 and 8 questions, respectively. Our results showed significant improvement in both symptoms $(P<0.001)$ and functional status $(P=0.013)$ components of $\mathrm{BQ}$ compared between the two visits (Table 1). FiftySix percent of the patients experienced an improvement in symptoms, while $68 \%$ were found to have enhanced functional status.

To evaluate the patients according to EDX results, patients were categorized into two groups regarding their first SNAPs findings. Patients with detectable SNAPs were included in the first group, while those with very low amplitude (less than $5 \mu \mathrm{v}$ ) and absent median SNAPs were placed in the second group. In the first group, all parameters except sensory distal latency and sensory proximal amplitude revealed a significant enhancement 1 year after the diagnosis of CTS (Table 2).

In the second group, 26 hands (63\%) were present in which 22 had absent SNAP (and 4 had very low amplitude). Of those with absent SNAP in the first visit, it becomes detectable in 14 (63\%) (significant but still in the severe CTS category), while the other $37 \%$ showed no response. However, 4 hands (16\%) with very low ampli- 
Table 1. Boston questionnaire variable after 1 year

\begin{tabular}{|c|c|c|c|}
\hline \multirow{2}{*}{ Variable } & \multicolumn{2}{|c|}{ Mean $\pm S D$} & \multirow[t]{2}{*}{$\mathbf{P}$} \\
\hline & Baseline & After 1 Year & \\
\hline Symptom in Boston questionnaire & $3.09 \pm 0.74$ & $2.77 \pm 0.81$ & $<0.001$ \\
\hline Function in Boston questionnaire & $2.35 \pm 0.60$ & $2.14 \pm 0.65$ & 0.013 \\
\hline
\end{tabular}

Table 2. Electrodiagnosis variable in the first group after a year

\begin{tabular}{|c|c|c|c|c|}
\hline \multirow{2}{*}{ Variable } & \multicolumn{2}{|c|}{ Mean \pm SD } & \multirow{2}{*}{ Mean Diff $\pm \mathrm{Cl}$} & \multirow{2}{*}{$\mathbf{P}$} \\
\hline & Baseline & After 1 year & & \\
\hline SDL & $1.97 \pm 0.28$ & $2.05 \pm 0.27$ & $0.08 \pm 0.13$ & 0.282 \\
\hline SD amp & $13.4 \pm 7.8$ & $8.05 \pm 2.7$ & $5.35 \pm 2.77$ & 0.004 \\
\hline $\mathrm{NCV}$ & $22.01 \pm 2.9$ & $25.2 \pm 7.2$ & $3.19 \pm 2.61$ & 0.006 \\
\hline $\mathrm{SPL}$ & $5.37 \pm 0.63$ & $5.01 \pm 0.80$ & $0.36 \pm 0.34$ & 0.003 \\
\hline $\mathrm{SP}$ amp & $15.10 \pm 9.3$ & $11.75 \pm 6.37$ & $3.35 \pm 3.78$ & 0.075 \\
\hline$M L$ & $5.33 \pm 0.78$ & $4.94 \pm 0.73$ & $0.39 \pm 0.35$ & 0.010 \\
\hline M amp & $7.55 \pm 3.38$ & $5.55 \pm 2.62$ & $2 \pm 1.43$ & 0.002 \\
\hline
\end{tabular}

SD: Standard Deviation; Cl: 95\% confidential interval; SDL: Sensory Distal Latency; SD amp: Sensory Distal Amplitude; NCV: Nerve Conduction Velocity; SPL: Sensory Proximal Latency; SP amp: Sensory Proximal Amplitude; ML: Motor Latency, M amp: Motor Amplitude

Table 3. Electrodiagnosis variable in the second group after a year

\begin{tabular}{|c|c|c|c|c|}
\hline \multirow{2}{*}{ Variable } & \multicolumn{2}{|c|}{ Mean $\pm S D$} & \multirow[t]{2}{*}{ Mean Diff $\pm \mathrm{Cl}$} & \multirow[t]{2}{*}{$\mathbf{P}$} \\
\hline & Baseline & After 1 Year & & \\
\hline SDL & $4.08 \pm 0.94$ & $3.50 \pm 1.43$ & $0.58 \pm 0.65$ & 0.109 \\
\hline SD amp & $1.51 \pm 4.28$ & $8.49 \pm 21.96$ & $6.98 \pm 5.39$ & 0.141 \\
\hline NCV & $13.06 \pm 3.22$ & $16.65 \pm 6.77$ & $3.61 \pm 2.89$ & 0.072 \\
\hline $\mathrm{SPL}$ & $7.55 \pm 1.06$ & $6.53 \pm 1.47$ & $1.02 \pm 0.69$ & 0.005 \\
\hline SP amp & $1.03 \pm 4.01$ & $5.79 \pm 6.52$ & $4.76 \pm 2.95$ & 0.003 \\
\hline$M L$ & $6.48 \pm 1.62$ & $6.20 \pm 1.48$ & $0.28 \pm 1.12$ & 0.455 \\
\hline M amp & $5.55 \pm 3.14$ & $4.90 \pm 2.52$ & $0.65 \pm 1.54$ & 0.203 \\
\hline
\end{tabular}

SD: Standard Deviation; Cl: 95\% confidential interval; SDL: Sensory Distal Latency; SD amp: Sensory Distal Amplitude; NCV: Nerve Conduction Velocity; SPL: Sensory Proximal Latency; SP amp: Sensory Proximal Amplitude; ML: Motor Latency; M amp: Motor Amplitude 
tude SNAP in the first visit developed no improvement in the second visit. In this group, only the sensory proximal latency $(\mathrm{P}=0.005)$ and sensory proximal amplitude $(0.003)$ had significant improvements in the follow-up EDX (Table 3).

In all patients, SPL, Motor Latency (ML), motor amplitude and sensory Nerve Conduction Velocity (NCV) had statistically significant enhancement.

Twenty-six hands of the patients participating in the study did not have any conservative treatment (45\%), and 22 benefited from splinting during the night (37\%). Other therapeutic modalities include physiotherapy, steroid injection, acupuncture, and the combination of physiotherapy and splinting.

Comparing those who had no treatment and splinting, symptoms improved significantly in both, but enhancement of functional status was only seen in the splinting group. In EDX of the patients without treatment, only SPL and MA improved significantly, while in the patients who applied night splints, Sensory Distal Latency (SDL), Sensory Distal Amplitude (SDA), SPL, ML, and NCV presented a statistically meaningful improvement.

\section{Discussion}

Several studies have proved a superior benefit of surgical intervention to conservative management in the treatment of CTS, in terms of symptoms and function. The patients undergoing surgical decompression were two times more likely to regain normal measures in nerve conduction studies but they may also suffer surgical complications as well. Given the potential and differential for adverse events in both therapeutic modalities, and that conservative interventions benefitted a substantial proportion of patients, the evidence supports the current practice of a trial of conservative management with surgical release for severe or persistent symptoms [11].

However, many patients with severe CTS abstain surgical interventions due to underlying medical conditions, surgical costs, surgical complications, etc. In this study, we aimed to investigate the functional scale and electrodiagnostic parameters after 1 year in non-operated patients with severe CTS.

Hui et al. demonstrated that open carpal tunnel release resulted in better symptomatic and neurophysiologic outcomes compared with steroid injection after a 20-week follow-up. However both treatments show improvement in electrodiagnostic parameters and functional scale of the studied groups after 20 weeks compared to the baseline [12].

A clinical trial by Gurcay Ag and his colleagues shows the equivalent effect of steroid injection and surgical decompression in the improvement of clinical and electrophysiological parameters in a short-term period in patients with severe CTS. They indicated that local steroid injection is a viable option in patients for whom surgical decompression is contraindicated or undesirable [13]

Demirci et al. found that the patients who received local steroid injection had improvement in NCV compared with those who underwent surgery in 3-6 months follow-up, but this improvement did not last long in the local steroid injection group. They showed that SNAPs latency, stimulating from the wrist, decreased significantly after 6 months. However, the decrement in the surgery group was statistically greater than the steroid injection group [5]. We found that sensory proximal latency was decreased significantly $(\mathrm{P}<0.001$ and $0.4895 \% \mathrm{Cl})$. We noticed that in both groups, those who used splint and the ones who did not have any treatment, the SPL improved greatly $(\mathrm{P}<0.05)$.

In our study, sensory conduction velocity improved significantly $(P=0.001)$, after a year especially in the group with recordable SNAPs in the first visits. We also detected the appearance of SNAP in the one-year EDX in 63\% of patients with a primary absent SNAP.

Another study by Khosravi et al. compared the effectiveness of steroid injection versus steroid injection plus splinting in severe CTS. Their results showed significant improvement of functional scale, patient satisfaction, and nerve conduction parameters after 4 weeks in both groups [14].

Another study by Lee et al. demonstrated that although the SPL in severe cases decreased about $1 \mathrm{msec}$ after 2 months following steroid injection, the decrement was not statistically significant. Due to the unclear number of participants in the mentioned study, its results are unreliable to compare [3].

Distal motor latency decreased in the first group $(P<0.01)$, but in the group, with absent SNAPs it did not improve. Besides, motor latency had improved in cases applying splint $(\mathrm{P}=0.03)$. In other studies, $\mathrm{DML}$ improved significantly at the follow-up after conservative treatments $[5,13,15]$. In addition, Demirci et al. and Gerritsen et al. showed that the improvement was not statistically significant between the conservative 
and surgery groups $[5,16]$. This could be due to severe motor axon loss in the severe CTS which could not even be regenerated properly after surgery, too. Lee et al. study did not show any great improvement in the second month after steroid injection. As mentioned above, it could be due to the low number of cases.

The symptoms and functional status improved significantly, though the symptoms got better greatly. The data of other researches were mostly in favor of our results $[3,5,7,13,14,16,17]$. Our study showed that patients who used splint had meaningful enhancement in both the symptoms and functional scores, while the others who had no treatment only showed enhancement in their symptoms. The different results in these 2 groups may be due to the improvement of neural impairment that affected the pain perception.

The current study is genuine in following non-operated patients with severe CTS for 1 year in an outpatient setting, for which no similar studies are available as a historical cohort.

One of the limitations of this study is that too many individuals lost to follow-up due to changes in address and phone number and their unwillingness to continue the study which is quite foreseeable in studies performed in an outpatient setting. We solved this issue by the enrollment of twice the calculated sample size of patients, and the 60 hands in 39 individuals showed the $80 \%$ power in reporting the results which is appropriate for statistical analysis. However, due to small numbers in subgroups of conservative treatment, the comparison could not be performed between the therapeutic measures.

Another limitation was the sampling bias of non-operated patients who may share the same characteristics which may affect their attitude in answering the questionnaire; this limitation was solved by adding quantitative values of EDX parameters that are not amenable to bias.

EDX measurements are quantitative measures that are not subject to biases; however, reporting EDX results by the senior author (AA) in both sessions provides better reliability and validity recordings.

Like any other before-after research studies, the bias of the examiner should be considered as a limitation which is based on previous experience, judgment, and evidence of the examiner. Blinding the examiner to the outcome measures will avoid such bias. However, due to the nature of EDX as a test based on physics and elec- trical conduction, as well as avoiding several examiners which might affect inter-rater reliability, both before and after EDX studies were performed by 1 person (AA). A similar study with a blinded examiner will be helpful to eliminate such bias.

\section{Conclusion}

Patients with severe CTS have substantial chances of improvement with conservative treatments, and surgery can be deferred if median SNAPs are detectable in their electrodiagnostic tests. However, surgery should be considered early in the management of patients with absent median SNAPs as conservative measures rarely provide relief.

\section{Ethical Considerations}

\section{Compliance with ethical guidelines}

The written informed consent forms and intervention method mentioned in the method section were reviewed and approved by the Institutional Review Board Members of the Ethical Committee at the vice chancellor for research of Shiraz University of Medical Sciences, Iran (IR.SUMS. REC.1391.4126).

\section{Funding}

The present paper was extracted from the MD. thesis by Zahra Hooshanginezhad at Department of Physical Medicine, Rehabilitation Shiraz University of Medical Sciences, Shiraz.

\section{Authors' contributions}

Conceptualization and design: Alireza Ashraf, Attiyeh Vasaghi; Data collection: Zahra Hooshanginezhad; Drafting the article: Attiyeh Vasaghi, Nima Derakhshan; Critically revising the article: Nima Derakhshan, Alireza Ashraf; Reviewing submitted version of manuscript: Nima Derakhshan; Approving the final version of the manuscript: Alireza Ashraf, Attiyeh Vasaghi, Zahra Hooshanginezhad, Nima Derakhshan.

\section{Conflict of interest}

The authors certify that they have no affiliations with or involvement in any organization or entity with any financial interest (such as honoraria; educational grants; participation in speakers' bureaus; membership, employment, consultancies, stock ownership, or other equity interest; and expert testimony or patent-licensing arrangements), or 
non-financial interest (such as personal or professional relationships, affiliations, knowledge or beliefs) in the subject matter or materials discussed in this manuscript.

\section{References}

[1] Tay LB, Urkude R, Verma KK. Clinical profile, electrodiagnosis and outcome in patients with carpal tunnel syndrome: A Singapore perspective. Singapore Medical Journal. 2006; 47(12):1049-52. [PMID]

[2] Ghasemi-Rad M, Nosair E, Vegh A, Mohammadi A, Akkad A, Lesha $E$, et al. A handy review of carpal tunnel syndrome: From anatomy to diagnosis and treatment. World Journal of Radiology. 2014; 6(6):284-300. [DOI:10.4329/wjr.v6.i6.284] [PMID] [PMCID]

[3] Lee JH, An JH, Lee SH, Hwang EY. Effectiveness of steroid injection in treating patients with moderate and severe degree of carpal tunnel syndrome measured by clinical and electrodiagnostic assessment. The Clinical Journal of Pain. 2009; 25(2):111-5. [DOI:10.1097/AJP.0b013e3181847a19] [PMID]

[4] Mav pen carpal tunnel surgery: avoiding the pitfalls. European Journal of Orthopaedic Surgery \& Traumatology. 2009; 19(1):11-7. https://link.springer.com/article/10.1007/s00590-008-0368-8

[5] Demirci S, Kutluhan S, Koyuncuoglu RH, Kerman M, Heybeli N, Akkuş $\mathrm{S}$, et al. Comparison of open carpal tunnel release and local steroid treatment outcomes in idiopathic carpal tunnel syndrome. Rheumatology International. 2002; 22(1):33-7. [DOI:10.1007/ s00296-002-0184-0] [PMID]

[6] Shi Q, MacDermid JC. Is surgical intervention more effective than non-surgical treatment for carpal tunnel syndrome? A systematic review. Journal of Orthopaedic Surgery and Research. 2011; 6(1):1-9. [DOI:10.1186/1749-799X-6-17] [PMID] [PMCID]

[7] Andreu JL, Ly-Pen D, Millán I, de Blas G, Sánchez-Olaso A. Local injection versus surgery in carpal tunnel syndrome: neurophysiologic outcomes of a randomized clinical trial. Clinical Neurophysiology. 2014; 125(7):1479-84. [DOI:10.1016/j.clinph.2013.11.010] [PMID]

[8] Meirelles LM, Santos JB, Santos LL, Branco MA, Faloppa F, Leite VM, et al. Avaliação do questionário de Boston aplicado no pósoperatório tardio da síndrome do tunel do carpo operados pela técnica de retinaculótomo de paine por via palmar. Acta Ortopédica Brasileira. 2006; 14(3):126-32. [DOI:10.1590/S141378522006000300002]

[9] Rezazadeh A, Bakhtiary A H, Samaei A, Moghimi J. [Validity and reliability of the Persian Boston questionnaire in Iranian patients with carpal tunnel syndrome (Persian)]. Koomesh. 2014;15(2):138-145. http://koomeshjournal.semums.ac.ir/article-1-2101-fa.pdf

[10] Hassankhani GG, Moradi A, Birjandinejad A, Vahedi E, Kachooe $A R$, Ebrahimzadeh $\mathrm{MH}$. Translation and validation of the Persian version the Boston carpal tunnel syndrome questionnaire. Archives of Bone and Joint Surgery. 2018; 6(1):71-7. [DOI:10.22038/ ABJS.2017.22598.1587]

[11] Shi Q, MacDermid JC. Is surgical intervention more effective than non-surgical treatment for carpal tunnel syndrome? A systematic review. Journal of Orthopaedic Surgery and Research. 2011; 6(1):17. [DOI:10.1186/1749-799X-6-17] [PMID] [PMCID]

[12] Hui AC, Wong S, Leung CH, Tong P, Mok V, Poon D, et al. A randomized controlled trial of surgery vs steroid injection for carpal tun- nel syndrome. Neurology. 2005; 64(12):2074-8. [DOI:10.1212/01. WNL.0000169017.79374.93] [PMID]

[13] Gurcay AG, Karaahmet OZ, Gurcan O, Kazanci A, Karsli PB, Umay EK, et al. Comparison of short-term clinical and electrophysiological outcomes of local steroid injection and surgical decompression in the treatment of carpal tunnel syndrome. Turk Neurosurg. 2017; 27(3):447-52. [DOI:10.5137/1019-5149.JTN.15936-15.0]

[14] Khosrawi S, Emadi M, Mahmoodian AE. Effectiveness of splinting and splinting plus local steroid injection in severe carpal tunnel syndrome: A Randomized control clinical trial. Advanced Biomedical Research. 2016; 5:16. [DOI:10.4103/2277-9175.175902] [PMID] [PMCID]

[15] Todnem K, Lundemo G. Median nerve recovery in carpal tunnel syndrome. Muscle \& Nerve. 2000; 23(10):1555-60. [DOI:10.1002/1097-4598(200010)23:103.0.CO;2-Z]

[16] Gerritsen AA, De Vet HC, Scholten RJ, Bertelsmann FW, De Krom MC, Bouter LM. Splinting vs surgery in the treatment of carpal tunnel syndrome: A randomized controlled trial. The Journal of the American Medical Association. 2002; 288(10):1245-51. [DOI:10.1001/jama.288.10.1245] [PMID]

[17] Karl JW, Gancarczyk SM, Strauch RJ. Complications of carpal tunnel release. Orthopedic Clinics of North America. 2016; 47(2):425-33 [DOI:10.1016/j.ocl.2015.09.015] [PMID] 
This Page Intentionally Left Blank 